Review

\title{
Insights into the theranostic value of precision medicine on advanced radiotherapy to breast cancer
}

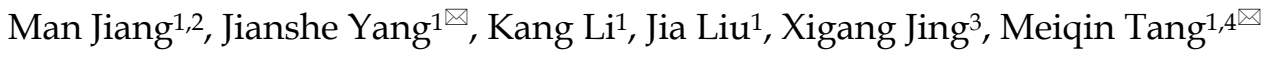 \\ 1. $3^{\text {rd }}$ Affiliated Hospital of the Chinese University of Hong Kong (Shenzhen), Shenzhen 518172, China. \\ 2. Department of Oncology, Longgang District People's Hospital, Shenzhen 518172, China. \\ 3. Medical College of Wisconsin (Milwaukee), Wisconsin 53226, USA. \\ 4. Department of Hematology, Longgang District People's Hospital, Shenzhen 518172, China. \\ $\triangle$ Corresponding authors: Professor Jianshe Yang, PhD, 3rd Affiliated Hospital of the Chinese University of Hong Kong (Shenzhen), No.53, Aixin Road, Shenzhen 518172, \\ China. Phone (Fax):+86-755-28932321, E-mail: yangj@@impcas.ac.cn; and Dr Meiqin Tang, Department of Hematology, Longgang District People's Hospital, Shenzhen \\ 518172, China, E-mail: tmq1203@163.com. \\ (C) The author(s). This is an open access article distributed under the terms of the Creative Commons Attribution License (https://creativecommons.org/licenses/by/4.0/). \\ See http://ivyspring.com/terms for full terms and conditions.
}

Received: 2020.06.23; Accepted: 2020.08.06; Published: 2021.01.01

\begin{abstract}
Breast cancer is the most common cancer in women worldwide. "Breast cancer" encompasses a broad spectrum of diseases (i.e., subtypes) with significant epidemiological, clinical, and biological heterogeneity. Each of these subtypes has a different natural history and prognostic profile. Although tumour staging (TNM classification) still provides valuable information in the overall management of breast cancer, the current reality is that clinicians must consider other biological and molecular factors that directly influence treatment decision-making, including extent of surgery, indication for chemotherapy, hormonal therapy, and even radiotherapy (and treatment volumes). The management of breast cancer has changed radically in the last 15 years due to significant advances in our understanding of these tumours. While these changes have been extremely positive in terms of surgical and systemic management, they have also created significant uncertainties concerning integration of local and locoregional radiotherapy into the therapeutic scheme. In parallel, radiotherapy itself has also experienced major advances. Beyond the evident technological advances, new radiobiological concepts have emerged, and genomic data and other patient-specific factors must now be integrated into individualized treatment approaches. In this context, "precision medicine" seeks to provide an answer to these open questions and uncertainties. Although precision medicine has been much discussed in the last five years or so, the concept remains somewhat ambiguous, and it often appear to be used as a "catch-all" term. The present review aims to clarify the meaning of this term and, more importantly, to critically evaluate the role and impact of precision medicine on breast cancer radiotherapy. Finally, we will discuss the current and future of precision medicine in radiotherapy.
\end{abstract}

Key words: breast cancer; radiotherapy; precision medicine; radiotherapy regime; radiosensitivity

\section{Introduction}

Breast cancer is the most commonly diagnosed cancer in women worldwide [1]. However, "breast cancer" is not, histologically-speaking, a homogeneous disease that can be easily classified according to prognostic criteria such as local, regional or distant disease extension (e.g., anatomical criteria). Rather, it is a complex pathology with a multifactorial etiology, and is highly heterogeneous in terms of its histological, molecular, and genomic characteristics [2]. This heterogeneity poses a major challenge to determining the optimal therapeutic sequence, particularly given the significant advances - in the span of only a few years - in classification systems. Until relatively recently, the staging was strictly based on anatomical disease extension, whereas it now includes conventional biomarkers such as estrogen receptors (ER), progesterone receptors (PR), HER2, ki-67, and histological grade (Table 1), as well as the molecular and gene profile of the tumour itself. Moreover, ongoing advance in genomic analysis techniques, which are gradually being validated and incorporated into clinical decision-making, has led to a reclassification of breast tumours based on their intrinsic biological characteristics. As a result, breast cancer is now recognized as a set of different diseases, each with a specific profile in terms of prognosis and treatment response, as shown in Figure 1 [3]. 
A

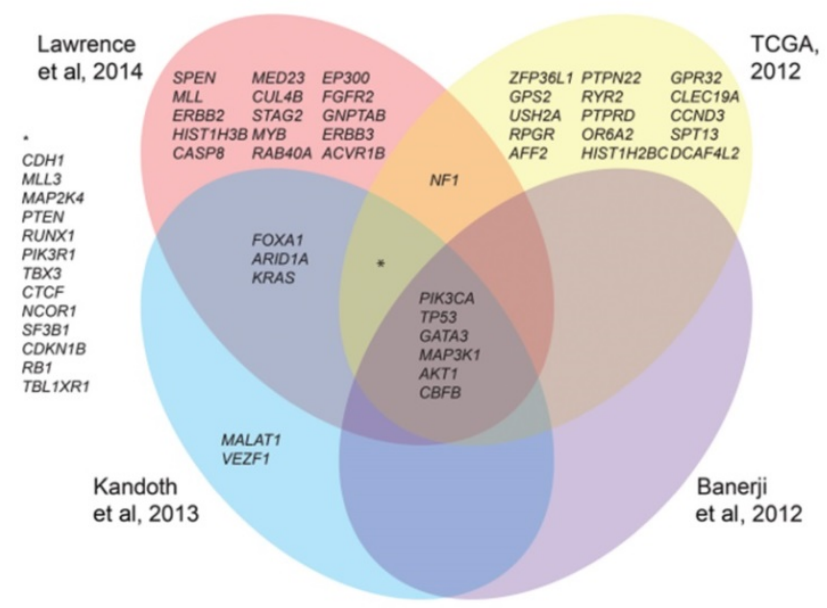

B

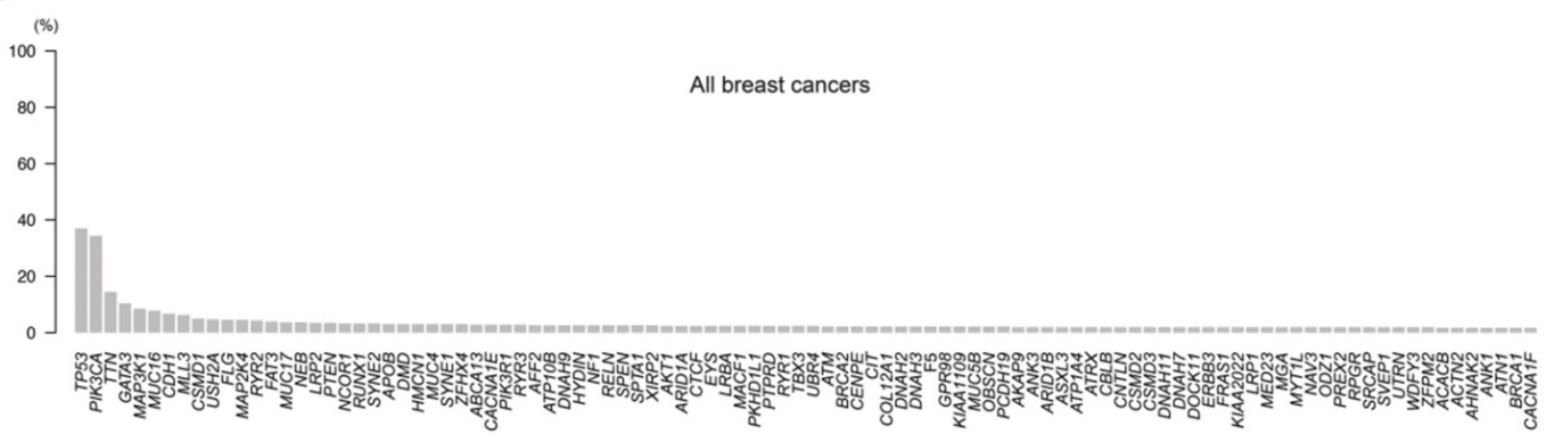

C
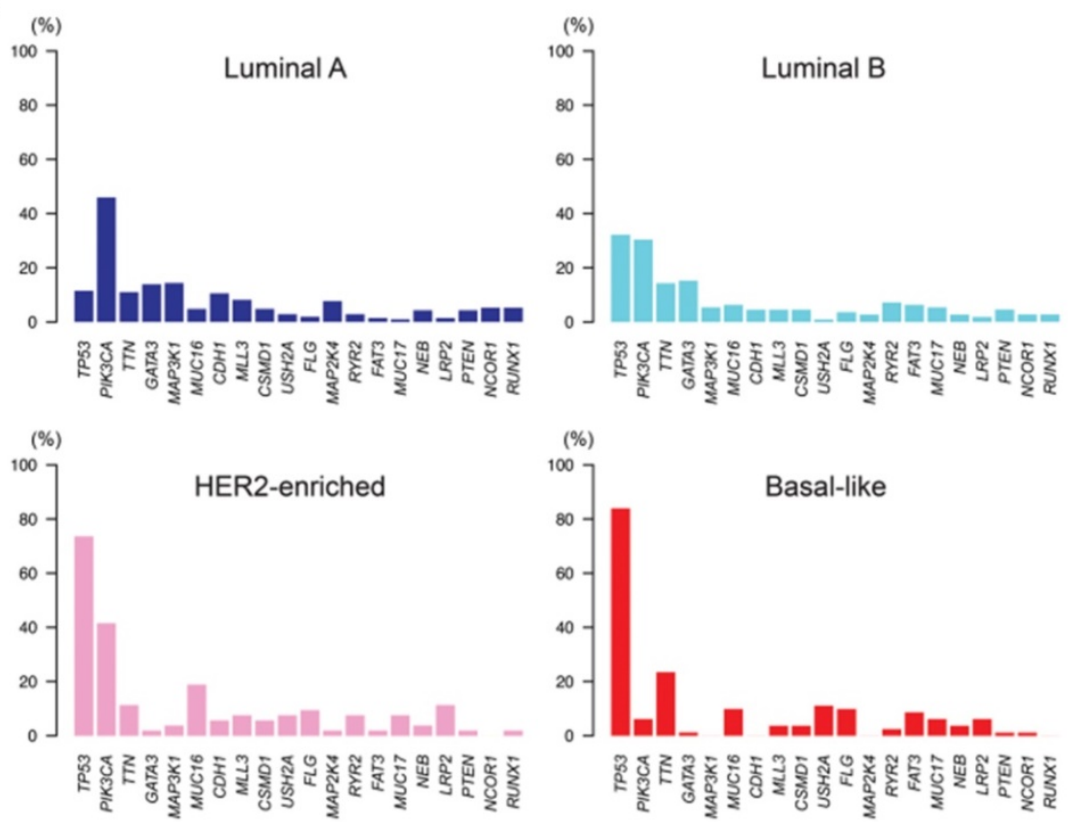

Figure 1. Intertumor genetic heterogeneity in breast cancer. At the genomic level, breast cancers are remarkably heterogeneous and no two tumors display an identical constellation of somatic mutations. A) Venn diagram illustrates the significantly mutated genes in breast cancer identified in different sequencing studies. B) Mutational frequencies of the 100 most frequently mutated genes in all breast cancers, illustrating the small number of genes highly recurrently mutated and a long "tail" of genes with low mutational frequency. C) The mutational frequencies of the 20 most frequently mutated genes in breast cancers of luminal A, luminal B, HER2-enriched and basal-like "intrinsic" subtypes. TCGA: The Cancer Genome Atlas [3].

The emergence of multigenic molecular assays (or genetic platforms) in recent years has revolutionized the management of breast cancer [4]. Initially, these assays were designed to provide prognostic and predictive data on response to chemotherapy in patients with positive hormone receptor expression and negative lymph nodes. However, over time, findings from validation studies have increasingly supported assay use in patients with positive nodes and low burden regional disease. 
Those same studies have also validated the capacity of these assays to predict the likelihood that a patient will benefit from chemotherapy [5], findings that have directly led to a reduction in the use of chemotherapy in low and intermediate-risk patients. These multiple developments now allow clinicians to determine which patients should receive systemic treatment more accurately. However, the impact on radiotherapy remains undefined, at least until results are reported from validation studies currently underway.

Table 1. Molecular subtypes of breast cancer

\begin{tabular}{|c|c|c|c|c|}
\hline Subtype & & Expression & Prognosis & Frequency \\
\hline \multirow[t]{2}{*}{ Luminal } & Luminal A & $\begin{array}{l}\text { ER+, PR -, HER2 -, } \\
\text { ki } 67 \leq 14\end{array}$ & Favourable & $60 \%$ \\
\hline & Luminal B & $\begin{array}{l}\text { ER+, PR +, HER2-, } \\
\text { ki67 > } 14\end{array}$ & Intermediate & \\
\hline $\begin{array}{l}\text { Basal-like } \\
\text { (triple } \\
\text { negative) }\end{array}$ & & $\begin{array}{l}\text { ER+, PR-, HER2- } \\
\text { ER-, PR-, HER2- }\end{array}$ & Poor & $15-20 \%$ \\
\hline HER2 + & & HER2+ & $\begin{array}{l}\text { Poor, but targeted } \\
\text { treatment is } \\
\text { available }\end{array}$ & $25 \%$ \\
\hline
\end{tabular}

ER: Estrogen receptors; PR: Progesterone receptors; HER2: Human epidermal receptor 2.

Adjuvant radiotherapy plays an essential role in the management of breast cancer, improving both locoregional control (preventing recurrences) and overall survival $[6,7]$. Notwithstanding these benefits, in recent years there has been a concerted effort to identify low-risk subgroups and subtypes with a good prognosis in which adjuvant radiotherapy could be omitted. These efforts are based on the premise that not all women benefit equally from radiotherapy, and that radiotherapy is unlikely to significantly improve survival in certain well-defined patient profiles $[8,9]$.

Moreover, the increasing use of neoadjuvant therapy, together with improved pathological response rates, suggests post-mastectomy radiation doses could be de-escalated or even omitted altogether [10]. This concept is directly related to recent efforts to define patient subgroups likely to benefit from the omission of a specific intervention, to prevent the associated acute or chronic side effects in patients who are unlikely to benefit (or only marginally benefit) from the treatment. The use of such treatments would be reserved for patients for whom there is a clear benefit. However, this approach overlooks the fact that we live in an era when, thanks to technological advances, it is possible to precisely deliver the radiation dose to the target volume while minimizing the dose to adjacent healthy organs and tissues. These characteristics of advanced, modern radiotherapy techniques also form part of "precision medicine", which is evident in two aspects of radiation oncology: 1) technologically-driven improvements in radiation delivery and 2) individualized treatment, with a personalized prescription for the dose, fractionation scheme, and target volumes based on the patient's clinical and biological parameters [11].

\section{Definition of the term "precision medicine"}

The term "precision medicine" has evolved from "personalized medicine", the much discussed "Holy Grail" of medicine. Personalized medicine is based on the determination of the unique genetic and molecular profile of an individual with a specific disease, data which can be used to develop a fully individualized treatment plan [12]. Over the years, particularly after the Human Genome Project was completed (due to the lack of immediate impact on health care), it became necessary to redefine the term "personalized medicine", perhaps because the term "personalized" proved to be not only too ambitious, but also too restrictive. This term fails to account for a substantial amount of relevant information, most notably clinical parameters and environmental factors. Currently, the concept of personalised medicine has been practically limited to molecular medicine. By contrast, the term "precision medicine" is a much broader term, which does not refer to the development of specific drugs for each individual, but rather to the adaptation of medical practice to suit the specific characteristics of well-defined groups of individuals, by classifying them into homogeneous subgroups based on their susceptibility to a particular disease, their probability of responding to a given treatment, or other parameters. In this way, any intervention - ranging from preventive to highly complex treatments - could be targeted at the specific subpopulations most likely to benefit from that intervention, resulting in the more efficient management of resources and even a reduction in systemic inequities $[13,14]$.

\section{Precision medicine applied to radiotherapy}

Radiotherapy is a local, targeted treatment modality that delivers highly precise conformal doses of radiation to the target in order to induce cell death. Tumours and healthy tissues both have an intrinsic radiosensitivity whose response is influenced by the total dose and the fractionation scheme [15]. Modern radiotherapy techniques can deliver conformal treatment with millimetric precision [16]. In addition, treatment response can be optimized by using radiosensitizers [17]. Radiotherapy may also play a 
role in modifying immune response [18], and some valuable bio-markers throughout the whole radiotherapy process were used to assess for evidence of toxicity, tumor recurrence or the development of the metastatic disease (Figure 2) [19]. By these ways, the difference of breast cancers, which routinely are considered as only one kind disease, will be distinguished systematically.

For all these reasons, radiotherapy could be one of the critical areas for precision medicine, and, as we discuss in this review, it is likely to become increasingly relevant in the treatment of cancer shortly. Radiotherapy is one of the three pillars of cancer therapy; the large number of cases treated with radiation therapy is more than sufficient to conduct studies to establish prognostic and/or predictive subgroups and to validate the results. Once we have identified these subgroups, the results could be applied in two ways: 1) precision radiotherapy based on imaging and clinical characteristics or 2) precision radiotherapy based on radiobiology and genomics [20]. The second point was discussed in-depth in this review.

\section{Genomically-guided radiotherapy}

As discussed above, breast cancer is not a homogeneous entity, but rather a spectrum of related diseases grouped under one umbrella term, with each biological subtype having its own natural history and prognosis. Indeed, the discovery of the biological diversity in breast cancer has led to the

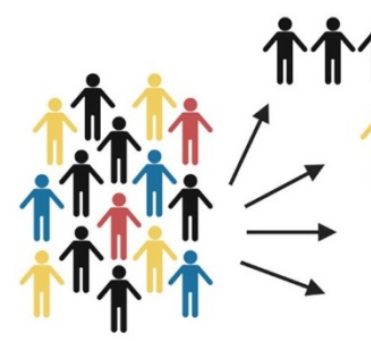

(1) Standiosensitive: stand treatment Highly radiosensitive: dose de-escalation

High risk of RT-induced toxicity: dose deescalation or alternative therapy

운 Radioresistant: dose escalation, radiosensitisers or alternative therapy

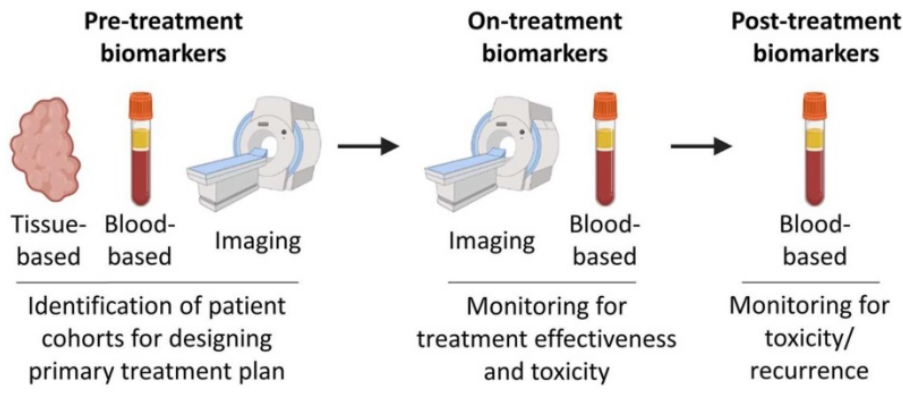

Figure 2. Precision medicine and radiotherapy. Patients could be stratified into different cohorts based on predicted intrinsic radiosensitivity and risk of toxicity. On-treatment monitoring may provide information on response to treatment, enabling adaptive changes to a patient's treatment to be made if necessary. Post-treatment biomarkers could be used to assess for evidence of toxicity, tumor recurrence or the development of metastatic disease [19]. re-stratification of patients into different prognostic groups. In turn, this has stimulated the development of systemic treatments directed at specific therapeutic targets, leading to improved survival and better control in appropriately selected patients. An excellent example of this is the development of anti-HER2 therapy in patients who overexpress this receptor. Over time, this biological subdivision of breast cancer has become increasingly relevant due to its predictive capacity, which can be used to predict the risk of metastatic progression and the probable impact of systemic treatment. Beyond this, multigene assays can also help to predict local or regional control [21].

Nguyen et al. [22] analyzed 793 breast cancer patients to evaluate the impact of molecular subtypes on local and regional control in patients treated with breast-conserving therapy followed by radiotherapy. Overall the probability of local recurrence in all subtypes was low, but particularly in the Luminal A subtype. Along the same lines, Caudle et al. [23] evaluated 595 patients treated with anthracycline and taxane-based neoadjuvant therapy followed by breast-conserving surgery (BCS) plus adjuvant radiotherapy. Although HER2+ patients were included, trastuzumab was not part of the neoadjuvant protocol. Locoregional recurrence (LRR)-free survival in patients with the HR+/HER2and HR+/HER2+ subtypes was excellent, regardless of tumour response to neoadjuvant chemotherapy; by contrast, patients with the HR-/HER2 + and HR-/HER2- subtypes who had a poor response to neoadjuvant treatment had a lower LRR-free survival rate, thus confirming a direct association between pathological complete response (pCR) and LRR-free survival. These findings are consistent with other reports, including the study by Guarnieri et al. [24] and the I-SPY1 multicenter trial [25], both of which found a higher rate of complete responses to neoadjuvant treatment in ER(-) patients. Guarneri and colleagues concluded that $\mathrm{pCR}$ could be considered a prognostic factor for overall survival (OS) while the I-SPY1 trial reached the same conclusion concerning relapse-free survival (RFS). The question, however, is how to integrate these findings into the adjuvant radiotherapy treatment plan effectively.

\section{Genomic assays for breast cancer}

Beyond the "histological" and immunohistochemical biomarkers 
conventionally used to classify patients into the subtypes described above, the real revolution that has taken place in recent years is the incorporation of genomic assays into routine clinical practice. These tests have redefined our understanding of the biology of breast cancer and its correlation with prognosis. Data from genomic assays even allow us to predict treatment response and the likely impact of different treatments.

These genomic tests are primarily used to assess the risk of progression, but a critical assessment of their applicability shows that they differ substantially in many ways, particularly concerning: the methodologies used to quantify gene expression; the genes evaluated; the clinical variables and pathologies included in the algorithm; risk group stratification; and whether testing is centralized or not. Therefore, even though these tests have been validated and proven to improve decision-making, it is important to keep in mind that two different assays, performed on the same patient, could yield contradictory results in terms of risk group assignment. [4] Nevertheless, these tests have demonstrated their clinical applicability, which has resulted in a dramatic decrease in chemotherapy, thereby reducing the cost of treatment and making it more cost-effective overall [26].

Numerous genomic tests are available, most commonly to identify patient subgroups in which adjuvant chemotherapy treatment can be omitted. In this review, we focus on the tests whose results could potentially influence the management of locoregional radiotherapy. Assays that do not address this question have been omitted from this review (Tables $2 \& 3)$.

Table 2. Overview of the multigene platform assays and implications for treatment decision-making

\begin{tabular}{|c|c|c|c|c|}
\hline & Oncotype DX & Oncotype DCIS & PAM50 & $\begin{array}{l}\text { Danish Breast Cancer } \\
\text { Cooperative Group }\end{array}$ \\
\hline Year & 2004 & 2013 & 2009 & 2014 \\
\hline No. genes used & 21 & 12 & 50 & 7 \\
\hline Method & RT-PCR & RT-PCR & RT-PCR & $\begin{array}{l}\text { Lasso method transferred to } \\
\text { RT-PCR }\end{array}$ \\
\hline N. risk groups & 3 & & 3 & 2 \\
\hline Tissue type used & FFPE tissue samples & FFPE tissue samples & FFPE tissue samples & FFPE tissue samples \\
\hline Risk categories & $\begin{array}{l}\text { Low } \\
\text { Intermediate } \\
\text { High }\end{array}$ & $\begin{array}{l}\text { Low } \\
\text { High }\end{array}$ & $\begin{array}{l}\text { Low } \\
\text { Intermediate } \\
\text { High }\end{array}$ & $\begin{array}{l}\text { Low } \\
\text { High }\end{array}$ \\
\hline $\begin{array}{l}\text { Inclusion of clinical } \\
\text { parameters }\end{array}$ & No & No & Yes & Yes \\
\hline $\begin{array}{l}\text { Indication for } \\
\text { Testinga }\end{array}$ & $\begin{array}{l}\text { ER+, HER2-, N- Estimates } \\
\text { chemotherapy benefit and } \\
\text { relapse risk during } \\
\text { hormonotherapy. }\end{array}$ & DCIS & $\begin{array}{l}\mathrm{ER}+/ \mathrm{N}-\text { and } \mathrm{N}+\text { treated by } \\
\text { hormonotherapy. Predicts } 10 \text {-year } \\
\text { metastasis-free survival. }\end{array}$ & $\begin{array}{l}\text { High risk breast cancer treated } \\
\text { with systemic therapy and } \\
\text { mastectomy. }\end{array}$ \\
\hline $\mathrm{ASCO} / \mathrm{NCCNa}$ & Yes (strong) & & Yes (moderate) & \\
\hline $\begin{array}{l}\text { Loco regional impact } \\
\text { of the test }\end{array}$ & $\begin{array}{l}\text { Predictor of loco regional } \\
\text { relapse }\end{array}$ & $\begin{array}{l}\text { Reveals the } 10 \text {-year risk of local recurrence; } \\
\text { Is significantly associated with risk of an } \\
\text { invasive local recurrence; } \\
\text { Is significantly associated with risk of a DCIS } \\
\text { local recurrence. }\end{array}$ & $\begin{array}{l}\text { Predictor of loco regional relapse } \\
\text { and local-regional free survival }\end{array}$ & $\begin{array}{l}\text { Predictor of the benefit of } \\
\text { radiotherapy }\end{array}$ \\
\hline $\begin{array}{l}\text { Potential application } \\
\text { in Radiotherapy }\end{array}$ & $\begin{array}{l}\text { Potential predictor of the } \\
\text { benefit of PMRT in high risk } \\
\text { patients. }\end{array}$ & $\begin{array}{l}\text { Omission of adjuvant radiotherapy after } \\
\text { conservative surgery in low risk DCIS } \\
\text { patients. }\end{array}$ & $\begin{array}{l}\text { Omission of adjuvant radiotherapy } \\
\text { after conservative surgery in low } \\
\text { risk invasive breast cancer patients. }\end{array}$ & $\begin{array}{l}\text { Potential predictor of the benefit } \\
\text { of PMRT in high risk patients. }\end{array}$ \\
\hline Validation studies & $\begin{array}{l}\text { NSABP B14 (retrospective) } \\
\text { NSABP B20 (retrospective) } \\
\text { SWOG } 8814 \text { (retrospective) } \\
\text { TransATAC (retrospective) } \\
\text { TAILORx (prospective) } \\
\text { RxPonder (prospective) }\end{array}$ & $\begin{array}{l}\text { ECOG E5194 (prospective-retrospective) } \\
\text { Ontario DCIS cohort (retrospective) }\end{array}$ & $\begin{array}{l}\text { ABCG8 (retrospective) } \\
\text { ATAC (retrospective) }\end{array}$ & DBCG- $82 \mathrm{bc}$ trial (retrospective) \\
\hline
\end{tabular}

Table 3. Gene panels studied in each genomic assay

\begin{tabular}{|c|c|c|c|c|c|c|c|c|}
\hline & & Institution & Platform used & Type of trial & Objective & $\begin{array}{l}\text { Study Start } \\
\text { Date }\end{array}$ & $\begin{array}{l}\text { Estimated } \\
\text { enrolment }\end{array}$ & $\begin{array}{l}\text { Estimated Study } \\
\text { Completion Date }\end{array}$ \\
\hline PRECISION & NCT02653755 & $\begin{array}{l}\text { Dana-Farber } \\
\text { Cancer Institute }\end{array}$ & Prosigna & $\begin{array}{l}\text { Interventional, } \\
\text { non-randomized } \\
\text { phase II clinical trial }\end{array}$ & Low risk score $=$ omission of $\mathrm{RT}$ & May 2016 & 690 & June 2023 \\
\hline TAILOR RT & NCT03488693 & $\begin{array}{l}\text { Canadian Cancer } \\
\text { Trials Group }\end{array}$ & Oncotype DX & $\begin{array}{l}\text { Interventional, } \\
\text { randomized phase } \\
\text { III clinical trial }\end{array}$ & $\begin{array}{l}\text { Regional radiotherapy in } \\
\text { Biomarker low risk } \\
\text { node-positive breast cancer }\end{array}$ & May 2018 & 2140 & December 2027 \\
\hline
\end{tabular}




\begin{tabular}{|c|c|c|c|c|c|c|c|c|}
\hline EXPERT & NCT02889874 & $\begin{array}{l}\text { Breast Cancer } \\
\text { Trials, Australia } \\
\text { and New Zealand }\end{array}$ & Prosigna & $\begin{array}{l}\text { Interventional, } \\
\text { randomized phase } \\
\text { III clinical trial }\end{array}$ & $\begin{array}{l}\text { Omission of radiation therapy } \\
\text { in } R O R \leq 60\end{array}$ & August 2017 & 1167 & December 2023 \\
\hline RIGAIN & NCT04069884 & $\begin{array}{l}\text { Sun Yat-Sen } \\
\text { Memorial } \\
\text { Hospital of Sun } \\
\text { Yat-Sen University }\end{array}$ & RecurIndexa & $\begin{array}{l}\text { Interventional } \\
\text { (Clinical Trial) } \\
\text { Randomized Phase } \\
\text { III }\end{array}$ & $\begin{array}{l}\text { Avoidance of Regional Nodal } \\
\text { Irradiation for Node Positive } \\
\text { Breast Cancer }\end{array}$ & $\begin{array}{l}\text { Not yet } \\
\text { recruiting }\end{array}$ & 1834 & \\
\hline BIORISE & NCT03252717 & $\begin{array}{l}\text { Institut du Cancer } \\
\text { de } \\
\text { Montpellier-Val } \\
\text { d'Aurelle }\end{array}$ & $\begin{array}{l}\text { Panel of five } \\
\text { proteins: AK2- } \\
\text { IDH2-ANX1- } \\
\text { APEX1- HSC70 }\end{array}$ & $\begin{array}{l}\text { Interventional } \\
\text { (Clinical Trial) } \\
\text { Single Group } \\
\text { Assignment. }\end{array}$ & $\begin{array}{l}\text { To confirm the predictive value } \\
\text { of the panel for } \\
\text { radiation-induced late side } \\
\text { effects after BCS+RT }\end{array}$ & August 2014 & $\begin{array}{l}500 \\
\text { participants }\end{array}$ & August 2022 \\
\hline
\end{tabular}

Search made on clinicaltrials.gov based on the following keywords: Radiotherapy, Recruiting, Not yet.recruiting Studies, Breast Cancer, Studies with Female Participants, Adult and excluding trials that did not consider the performance of a molecular, genomic or protein test.

Abbreviations: RT, Radiotherapy; BCS, breast conserving surgery.

\section{Oncotype DX}

The Oncotype DX 21 genomic test (OncotypeDX, Genomic Health, CA) was one of the first clinically validated molecular tests to provide a risk stratification model that could predict the benefit of adjuvant chemotherapy in patients with breast cancer. It was initially validated for patients with positive hormone receptors (HR) and negative lymph nodes [27], and later for women with positive lymph nodes with low burden axillary disease [28]. The test analyses formalin-fixed and paraffin-embedded tumour tissue to determine the expression of 21 genes using real-time reverse polymerase chain transcription (RT-PCR). Of these 21 genes, 16 are cancer-related and five are reference genes for normalization. The 16 genes were selected from 250 candidate genes based on the correlation between gene expression and the risk of distant recurrence. Validation studies have shown that this test provides information - beyond the usual histological and clinical parameters (immunohistochemical, histological and TNM) - about the potential benefits of chemotherapy in a given patient, as well as predictive data about the risk of recurrence [29]. This genomic test is now widely used, with a growing body of evidence showing that its use has significantly reduced the prescription of chemotherapy in breast cancer patients; moreover, there has also been an increase in the number of patients whose test results indicated a high risk of recurrence - despite the presence of good prognostic factors such as postmenopausal status, positive HR status, and negative nodes [30].

Given that locoregional recurrence is a prognostic factor for distant failure, several authors have attempted to determine the association between the Oncotype Dx recurrence score and the risk of locoregional failure. The Mamounas group was one of the first to explore this approach. Those authors evaluated node-negative, ER+ patients from the NSABP trials (B-14 and B-20). Mamounas et al. [31] included 895 and 355 patients, respectively, from the B-14 trial who received adjuvant tamoxifen or placebo; they also included 424 patients from the B-20 trial who were treated with chemotherapy and tamoxifen. To determine the impact of the Oncotype DX score, they correlated the recurrence score with the time to locoregional recurrence, finding a statistically significant association between the recurrence score in patients with node-negative, ER+ breast cancer who received tamoxifen. This finding is similar to the known association between the recurrence score and distant failure in the same group of patients. The same association was confirmed in patients (regardless of age), who underwent mastectomy alone without adjuvant radiotherapy (which was not indicated), suggesting that patients with this clinical profile who have a high-risk recurrence score could benefit from adjuvant radiotherapy. Despite these promising results, the clinical applicability of OncotypeDx in this context is still pending validation.

\section{Oncotype DX Breast DCIS Score test}

Studies have shown that adjuvant radiotherapy after BCS in patients with ductal carcinoma in situ (DCIS) reduces the likelihood of local recurrence (both in situ and invasive) by 50\% [32]. However, a significant number of patients do not receive adjuvant radiotherapy - even though it is considered the standard of care - mainly due to treatment decisions based on subjective, non-evidence based clinical criteria. Moreover, in patients with DCIS, there may be subgroups suitable for breast-conserving therapy without adjuvant radiotherapy, although this is not currently an option under current treatment standards.

Oncotype DCIS is a prognostic test to estimate the risk of local recurrence. The platform combines genomic data with clinical parameters (age, tumour size, surgical margins, and multifocality). The hypothesis underlying the development of this platform is that the Oncotype DX recurrence score could provide the same information in patients diagnosed with DCIS. It was first clinically validated in formalin-fixed, paraffin-embedded histological archival specimen blocks, with or without an invasive component, obtained from patients with DCIS (treated with BCS without radiotherapy) included in 
the E5194 ECOG-ACRIN clinical trial [33]. Of the 670 randomized patients (DCIS grade [G] 1 or $\mathrm{G} 2 \leq 2.5 \mathrm{~cm}$ versus $\mathrm{G} 3<1 \mathrm{~cm}$, and minimum surgical margin of 3 $\mathrm{mm}$ ), tissue samples were available from 327 patients. In this sample, the expression of a panel of 12 genes (seven cancer-related genes and five reference genes) was determined. These 12 genes were originally identified in a previous study of five experimental datasets (none with samples from this trial), which was used as a validation cohort (i.e., not experimental). The 10-year risk of ipsilateral breast cancer for patients classified as low, intermediate, and high risk, was, respectively, $10.6 \%, 26.7 \%$, and $25.9 \%$ $(p=0.006)$. The risk of an invasive event at 10 years was $7 \%, 12.3 \%$ and $19.2 \%$, respectively $(p=0.003)$. On the multivariate analysis, the Oncotype DCIS recurrence score, tumour size, and postmenopausal status were all significantly associated with local recurrence of any type [34].

This assay was independently validated in a subgroup of 3,303 patients from the Canadian cohort study (Ontario) involving 3,762 patients diagnosed with pure DCIS; histological samples from $80 \%$ of patients were available for centralized review [35]. That study showed that the Oncotype DCIS recurrence score was significantly associated with the risk of ipsilateral breast recurrence in ER+ patients [hazard ratio (HR), 2.3; 95\% CI, 1.41-3.59; $p<0.001$ ] and in the full cohort (including both ER positive and negative patients, $95 \%$ of whom were $\mathrm{ER}+)(\mathrm{HR}=2.2$; 95\% CI, 1.43-3.22; $p<0.001)$. The 10-year risk of developing an ipsilateral breast event was, respectively, $12.7 \%, 33 \%$, and $27.8 \%$ for patients with low, intermediate, or high risk. The corresponding 10 -year risk of an invasive event was $8 \%, 20.9 \%$, and $15.5 \%$, respectively [36]. The oncological outcomes in these patients are worth highlighting. Two subgroups were evaluated: 571 patients who underwent BCS alone, and 689 who underwent BCS plus adjuvant radiotherapy. At a median follow up of 9.2 years, 100 local recurrences were registered (57 invasives, 44 DCIS) in the first group versus 86 in the latter group (adjuvant radiotherapy). These results are of interest because, not only was the recurrence rate lower in the group that received adjuvant radiotherapy, but those patients also presented clinical factors at diagnosis suggestive of a worse prognosis. On the multivariate analysis, a propensity-score analysis showed that the following factors were significantly associated with local recurrence: radiotherapy, age at diagnosis, tumour size, and multifocality. The Oncotype DX DCIS risk group classification, adjusted for these variables, was significantly associated with the risk of local recurrence; however, even though women with a high-risk score had a higher risk of local recurrence (both DCIS and invasive), and also a more significant absolute benefit from radiotherapy compared to the low-risk group, the model was not a statistically significant predictor of the benefit of radiotherapy [37].

Given these findings, it is clear it would be risky to omit adjuvant radiotherapy after BCS in patients with DCIS based exclusively on data provided by this genomic assay. Nevertheless, the DCIS score has altered clinical practice-even impacting recommendations for adjuvant radiotherapy in some cohorts [38]-although more robust data from clinical trials are needed to validate the role of this assay to guide radiotherapy administration in women with breast cancer (NCT02766881). In short, the impact of omitting adjuvant radiotherapy on oncological and clinical outcomes remains unknown at present. Nonetheless, it is important to emphasise that this assay was not designed, nor has it been validated, as a predictor of response to radiotherapy or hormone therapy. Moreover, the data reported to date have failed to identify a subgroup of patients who would not benefit from adjuvant radiotherapy after BCS. Finally, this genomic test has not been evaluated to determine the existence of high-risk DCIS subgroups that could benefit from post-mastectomy radiotherapy despite the presence of good prognostic clinical factors [39].

\section{Prosigna/PAM 50}

The classification of breast cancer according to the biological subtype has gained prominence in recent years. At the same time, due to technological innovations, it is now possible to identify transcriptional differences based on RT-PCR and microarray analyses, correlating biological subtypes with different transcriptional patterns. In the year 2000, Perou and colleagues classified, for the first time, breast cancer at the molecular level. Those authors hypothesised that phenotypic diversity in breast cancer was attributable to the diversity in gene expression patterns, which could be studied using cDNA microarrays [40,41]. They evaluated 65 surgically-resected specimens (normal and cancerous tissues) from 42 women with breast cancer who underwent neoadjuvant treatment. They identified 496 genes that presented more significant variability in terms of their expression among the tumours, but their expression was only minimally variable in the samples from the same patient (intrinsic genes). Based on these data, together with microarray and RT-PCR techniques, Parker et al. developed a simplified signature for a 50-gene subtype predictor (classifier) and five control genes that were strongly correlated with the biological subtypes [42] and superior to 
routine immunohistochemical studies [43]. This gene signature assay (PAM50) is marketed as the Prosigna-Breast Cancer Prognostic Gene Signature Assay. The algorithm resulting from the combination of genomic and clinical data (proliferative index, tumour size, nodal involvement) provides a Risk of Recurrence (ROR) score that assigns the patient to one of three risk categories (low, intermediate, high) associated with varying probabilities of developing a distant recurrence at 10 years. This assay was validated on patient samples from various clinical trials. An analysis of 1017 patients in the ATAC study showed that the PAM50 ROR in ER+, node-negative patients was more accurate than the OncotypeDx recurrence score in identifying high-risk patients, reducing the number of intermediate-risk patients [44]. Besides, an analysis of $1478 \mathrm{ER}+$ postmenopausal patients from the ABCSG-8 trial (tamoxifen vs tamoxifen followed by adjuvant anastrozole) showed that the ROR had a better prognostic capacity than conventional clinical parameters, leading the authors to conclude that adjuvant chemotherapy in low-risk patients did not provide any expected oncological benefits [45].

The PAM50 assay is also being studied to determine the impact of the different intrinsic biological subtypes on local recurrences, and to identify clinical cases or intrinsic subtypes in which adjuvant radiotherapy can be omitted. A secondary analysis of the ABSCG-8 trial examined the association between the ROR and the biological subtype in terms of LRR-free survival, concluding that the ROR was highly predictive of locoregional recurrence, regardless of nodal status, age, or tumour size [46]. That study included 1,308 patients, $79 \%$ of whom were treated with BCS plus adjuvant radiotherapy. At 10 years, a total of 34 locoregional recurrences were observed. A comparison of outcomes by biological subtype revealed a significant difference $(p=0.022)$ in survival free of local or nodal recurrence between luminal A $(98.1 \%)$ and luminal B (95.9\%). However, that study was not designed for decision-making for adjuvant radiotherapy, which provides an opportunity for other researchers to evaluate this question. The PRECISION (Profiling Early Breast Cancer for Radiotherapy Omission) trial is a non-randomized, phase II trial (NCT02653755), which began patient recruitment in 2016. That trial aims to evaluate the omission of breast radiotherapy action after lumpectomy in patients with low-risk (ROR score) breast cancer in whom adjuvant endocrine therapy is indicated. A total of 1,380 patients are expected to be included in that trial through the year 2023. Along the same lines, the EXPERT trial (Examining Personalised Radiotherapy for Low-risk Early Breast Cancer), with similar inclusion criteria and objectives, is being carried out by the Breast Cancer Trial Group in Australia and New Zealand (ANZ 1601/BIG16-02) to identify women (using Prosigna) with low-risk breast cancer. Recruitment is expected to be completed in 2022 [TROG 16.04, ANZ 1601/BIG16-02].

\section{Danish breast cancer cooperative group gene profile}

The study was based on the patient cohort included in the Danish Breast Cancer Cooperative Group (DBCG82bc) trial [47]. The objective was to validate a gene profile associated with the risk of locoregional recurrence in patients who did not receive adjuvant radiotherapy. A second aim was to determine if this profile could predict the benefit of radiotherapy in patients with high-risk breast cancer who received adjuvant systemic treatment and were randomized to receive or not postmastectomy radiotherapy. Gene expression analysis was performed on frozen tumour tissue samples obtained from 191 patients, all of whom underwent mastectomy. The genes were identified by the Lasso method, and the endpoint was locoregional recurrence (LRR). A weighted gene expression index (DBCG-RT profile) was calculated and transferred to quantitative real-time PCR (RT-PCR) in formalinfixed and paraffin-embedded samples (FFPE). Seven genes were identified; based on the DBCG-RT profile, the 191 patients were categorized as having either high or low risk of LRR. A "low risk" classification was consistently associated with biological subtypes (Luminal A) indicative of good prognosis and these patients also had better locoregional control; by contrast, the "high risk" category was correlated with triple negative and HER2 subtypes. Based on these findings, it is clear that this gene profile strongly identifies the same subtypes, with the same prognostic behavior, as if they had been determined by other methods (histological/immunohistochemical). Adjuvant radiotherapy after mastectomy significantly reduced the risk of recurrence in patients with a "high risk" of LRR, but not in "low risk" patients. Interestingly, both risk groups (high and low) were present in all clinicopathological subgroups, and their outcomes were independent of classic variables such as TNM. For example, among the patients considered to present a high risk of recurrence according to conventional criteria (tumour size $>5 \mathrm{~cm}$ in $\geq 4$ positive lymph nodes), some were considered to have a very low risk of LRR based on the DBCG-RT profile; conversely, some patients with low lymph node load or tumour size $<2 \mathrm{~cm}$ were classified as "high risk" (DBCG-RT profile), and radio- 
therapy was beneficial in those cases. These findings suggest that the DBCG-RT profile could add valuable information to help guide decision-making [48].

\section{The possibility of applying biology- guided radiation therapy}

In recent years, it seems that the vast majority of research has been centred on identifying patient subsets in which adjuvant radiotherapy can be safely omitted. In the coming years, it is certainly possible that a genomic assay capable of identifying this patient subset will be developed and validated. However, a reasonable criticism of the current role of biomarkers in radiation oncology is the lack of specific biomarkers to guide the indication for radiotherapy treatment itself. In general, biomarkers evaluated in the past were not selected (nor evaluated) for their specificity as indicators for radiotherapy. Indeed, most of the biomarkers shown to correlate with postradiotherapy treatment outcomes are also prognostic in patients who do not undergo radiotherapy. In this regard, rather than using genomics to identify biomarkers to guide the omission of radiotherapy in low-risk patients, the real benefit would be to use genomics to adapt the treatment approach to suit the intrinsic characteristics of the tumour itself.

Eschrich et al. developed and validated the radiation sensitivity index (RSI) as a predictor of the inherent radiosensitivity of a given tumour. This indicator was evaluated in different cell lines from different types of cancer (including breast cancer) [49], and validated on datasets from two independent centres, the Karolinska University Hospital $(n=159)$ and the Erasmus Medical Center $(\mathrm{n}=344)$. In the first cohort (Karolinska) [50], patients whose tumours were predicted to be radiosensitive had better 5-year RFS outcomes than those with radiation-resistant tumours ( $95 \%$ vs $75 \%, p=0.0212$ ), but there were no differences between patients who did not undergo radiotherapy ( $71 \%$ vs $77 \%, p=0.6744)$, thus validating RSI in this patient subset, a finding that indicates that RSI is specific to radiotherapy. In the Erasmus cohort, radiosensitive patients treated with radiotherapy had better 5-year metastasis-free survival than patients with radiation-resistant tumours $(77 \%$ vs $64 \%$, $p=0.0409$ ), with no differences in patients who did not receive RT ( $80 \%$ vs $81 \%, p=0.9425)$. The multivariate analysis showed that the variable that had the strongest influence on outcomes in radiotherapytreated patients was RSI, demonstrating that RSI is an independent predictor of outcomes in ER+ patients treated with radiotherapy. Subsequently, Sjöström et al. [51] developed and validated a gene expression assay to predict the risk of local breast cancer recurrence and its response to adjuvant radiotherapy after BCS. Torres-Roca and colleagues evaluated the possibility of integrating RSI with molecular subtype data to identify patient subgroups with a higher risk of local recurrence after BCS+ radiotherapy, finding that patients with the triple negative and radioresistant subtype had an increased risk of local recurrence. These findings are sure to motivate more research in this area. The authors have suggested that the combination of RSI and molecular subtype could help to guide indications for adjuvant radiation therapy in breast cancer [52].

In recent years, numerous authors have attempted to integrate data on the intrinsic radiosensitivity of tumours with the traditional linear quadratic model. The conventional model quantifies the biological effect of the radiation dose on both the tumour and healthy tissue, but this model has a significant limitation: it is not patient specific, and its value is reduced when extreme hypofractionation is used [53]. Integration would require developing a customized model using a patient-specific " $a$ " to quantify the response of a specific tumour to radiotherapy to obtain a genomic-adjusted dose (GARD) [54]. The premise underlying this model in breast cancer is that a significant number of radiosensitive women could be treated with a lower than standard dose without compromising local control. In addition, it could be possible to identify those patients in whom dose escalation would likely yield better results, thus offering "true" oncological personalization, as well as optimizing fractionation on a case by case basis [55]. Ahmed et al. evaluated the feasibility of combining the linear quadratic model with the RSI, calculating the GARD that would optimize the individualised radiation dose for patients from two cohorts with triple negative (TN) breast cancer. Those authors also demonstrated that the GARD, like the RIS, is associated with the risk of local recurrence. Thus, they identified two groups of patients based on the RIS value. One group (RIS, 0.43-0.49) would likely benefit from dose escalation to $50-60$ Gy (29\% of the sample) and a second group (RIS up to $0.55,13 \%$ of the sample) would benefit from higher doses (70Gy), in both cases with conventional fractionation schemes (2 Gy/session). Although the results of this hypothesis-generating study cannot be applied to routine clinical practice, these findings warrant a prospective clinical trial [56].

While the intrinsic radiosensitivity of the tumour is important, the radiosensitivity of healthy tissues is also relevant. In fact, many studies have explored the impact of radiation on healthy tissues and the likelihood of developing specific side effects. In breast cancer, manifestations of acute and chronic toxicity fibrosis, edema, telangiectasias, hyperpigmentation, 
and different degrees of dermitis - have been associated with clinical risk factors (tobacco use, body mass index, hypertension, skin quality prior to radiotherapy) [57] or with the radiotherapy treatment itself (outdated techniques, size of the volume irradiated, and unfavourable dose-volume histograms) [58]. However, treatment-related toxicity has not been eliminated, even in young patients with no relevant risk factors treated with state-of-the-art techniques such as hypofractionated IMRT or partial breast irradiation $[59,60]$. For healthy tissues - at least in some organs - the gene profile could influence up to $80 \%$ of radiosensitivity and, therefore, the associated toxicity [61]. That said, validation studies are still lacking in this field, and most of the studies published to date present methodological deficiencies, which limits reproducibility [62]. Genome-wide association studies (GWAS) have been conducted to identify common breast cancer susceptibility alleles. These studies seek to expand the search for specific candidate genes to the study of a large number of single-nucleotide polymorphisms (SNP) in genes that encode proteins involved in pathways associated with inflammatory processes in tissues and with DNA damage, to find consistent differences between these SNPs in order to determine the association between these SNPs and radiation side effects [63]. Conceivably, if a large number of SNPs can be identified, then it would be possible to establish a polygenic risk score that could subsequently be applied in routine clinical practice [64]. GWAS technology is based on genotyping platforms (chip-based microarray technology) that can evaluate hundreds to thousands of SNPs simultaneously. However, to apply this in clinical practice, well-structured and homogeneous clinical data are needed to avoid bias [65], but this is difficult given that most analyses have been conducted in small cohorts, which substantially lowers the statistical power [66]. Even though some published studies have confirmed these associations, few have been independently validated and nearly all suffer from a limited " $n$ " [67]. International collaborative groups have been formed to expand our understanding of the genomic basis for differences in radiosensitivity [68] by generating large, well-structured datasets that can be evaluated and associated with clinical characteristics [69], as well as to establish uniform working protocols (methodologies) and to jointly communicate the results of these genetic association studies in radiogenomics [70].

In recent years, there has been a geometric increase in the number of published studies on breast cancer. Unfortunately, the quality of the evidence is limited. Consequently, we must remain cautious, avoiding categorical statements, and proceeding carefully when seeking to apply discoveries into routine clinical practice. Nonetheless, several interesting studies have been published in recent years, and the promising results reported in those studies open the door to further research in this field. Mutations in the ATM (ataxia-telangiectasia mutated) gene were among the first to be evaluated for their possible association with tissue radiosensitivity. The ATM gene plays an important role in response to ionizing radiation and it is involved in the detection of DNA double-strand breaks and the initiation of pathways leading to the arrest of the DNA repair cycle or apoptosis [71]. It has been shown that patients with a truncated mutation in both copies of the ATM gene are likely to develop significant radiationinduced toxicity, and it is precise that these patients who develop ataxia-telangiectasia syndrome [72]. The SNP rs1801516 (c.5557G> A, p.Asp1853Asn) is among the ATM SNPs that have been most thoroughly investigated. Its impact on radiationinduced toxicity has been evaluated in three metaanalyses, with conflicting results: two of those studies found a direct association with an increased probability of fibrosis [73] and acute toxicity [74], but the third did not identify any consistent associations [75]. Andreassen et al. [76], on behalf of the International Radiogenomics Consortium, conducted an individual patient data meta-analysis to assess the relationship between the ATM rs1801516 SNP and toxicity after radiotherapy in 5456 breast and prostate cancer patients from 17 different cohorts (breast cancer, $n=2759$; prostate cancer, $n=2697$ ), concluding that there is an association between the ATM rs1801516 Asn allele and increased risk of radiationinduced toxicity (odds ratio of approximately 1.5 for acute toxicity and 1.2 for late toxicity). These findings warrant future large studies in more homogeneous cohorts. Studies in smaller cohorts have also attempted to find associations between SNPs and post-radiotherapy pain [77], skin toxicity [78], and fibrosis [79], but the results have not been validated and further studies are required.

\section{Chances of precision medicine in breast cancer radiotherapy}

Until just a few years ago, radiotherapy treatment in breast cancer was based on clinical parameters, in which the following questions were crucial: What is the size of the tumour? Is the patient node positive or not? Are the surgical margins negative? Mastectomy or BCS? Will the patient benefit from a boost or not? The responses to these questions determined the indication (or not) for radiotherapy, 
and also determined target volume selection and the scheme, usually $50 \mathrm{~Gy}$ in 25 sessions of $2 \mathrm{~Gy}$. However, about 15 years ago, research efforts began to look beyond these basic questions. In parallel with technological advancements and an improved understanding of the radiobiology of both cancerous and healthy tissue, hypofractionated protocols began to be tested, generally by reducing the number of fractions from 25-30 sessions (the traditional scheme) to only 15-20 sessions. These approaches were evaluated in high quality, methodologically-sound randomized clinical trials, which confirmed the therapeutic equivalence of these hypofractionated protocols with conventional protocols, but with several potential advantages, including reduced costs, improved QoL, and less objective toxicity [80]. From there, the next logical step was to propose partial breast irradiation protocols [81] and the omission of radiotherapy in selected patients [82]. This began a period in which the focus shifted to treatment de-escalation, the concept of "less is more", which evolved in parallel with surgical de-escalation, during which radical surgery (e.g., Halsted mastectomy) was abandoned in favour of less radical approaches, eventually leading to oncoplastic surgery [83]. Furthermore, thanks to the development of new drugs and systemic treatments that have substantially improved locoregional tumour response, adjuvant radiotherapy is now even considered in certain node positive patients who respond to neoadjuvant chemotherapy [84]. However, it is important to keep in mind that all these advances are solely and exclusively based on clinical parameters, without consideration of the intrinsic biological characteristics present in individual tumours. In addition, these advances also ignore all of the new instruments capable of estimating the risk of recurrence and survival, as well as tools that use genomic data to estimate radiosensitivity and toxicity.

Although we are making progress (Table 4), this research is still in its infancy. Molecular signatures and radiosensitivity indices are still in the early stages of development, with limited clinical applicability due to the lack of validation in patient cohorts without selection biases (most validation studies have been performed on non-randomized datasets with numerous confounders). Moreover, currently available genomic assays to predict recurrence risk were not designed - and thus not validated to personalize radiotherapy (i.e., to determine whether radiotherapy is indicated or not and to define the target irradiation volumes) [85].

\section{Conclusions}

Beyond the obvious technological and clinical advances (e.g., fractionation protocols) that have become standard practice in recent years, the true promise of precision radiotherapy in breast cancer is the integration of clinical and imaging data together with molecular data and genomic markers. This is expected to lead to the development of numerous prognostic and predictive instruments to help predict various outcomes, most importantly: a) treatment response based on the intrinsic radiosensitivity of the tumour, thus allowing for dose modification; $b$ ) the risk of locoregional recurrence, which could be used to guide target volume modifications; and c) the risk of acute or chronic toxicity, which would allow us to modify the treatment to avoid these undesired effects, or at least initiate early treatment, thus improving tolerance to radiotherapy.

Table 4. Open clinical trials underway to evaluate the impact of data provided by gene or protein panels on radiotherapy treatment

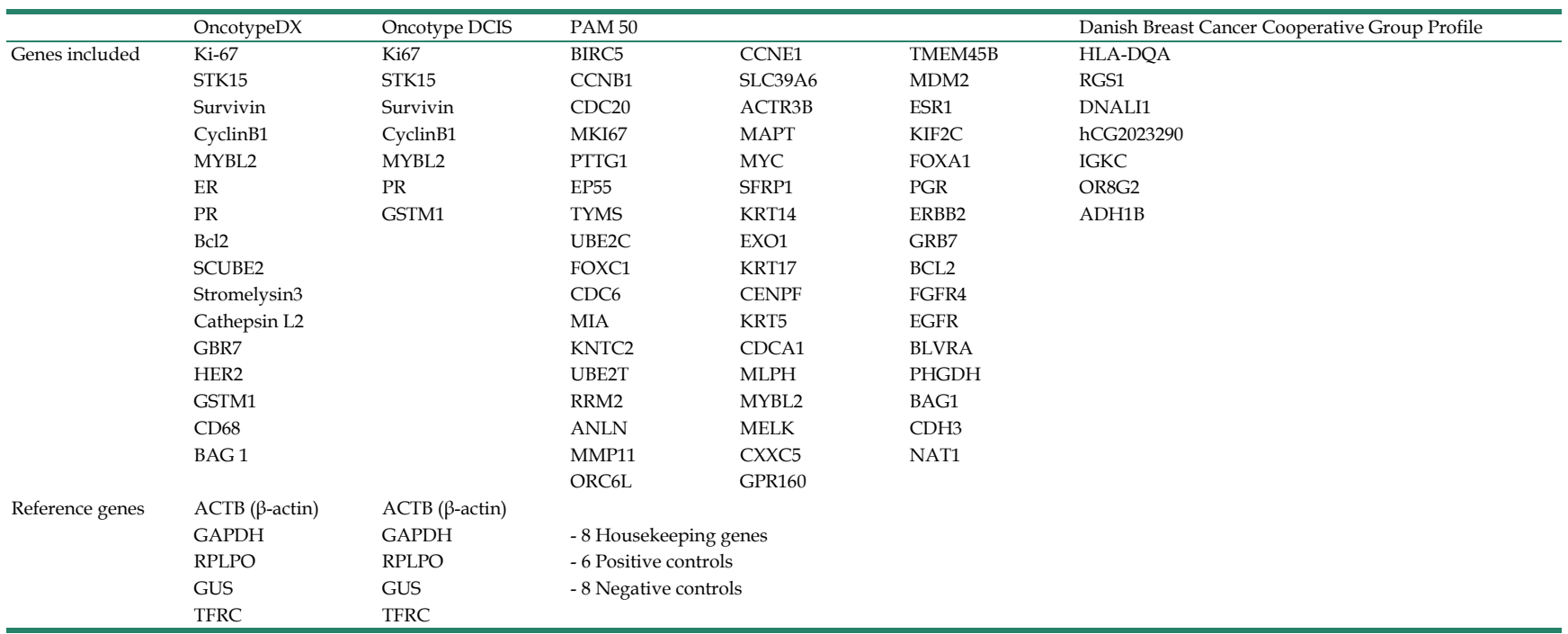


At present, several uncertainties remain. The underlying mechanisms of individual radiosensitivity remain unclear, molecular assays that could potentially play a key role in radiotherapy decisionmaking still need to be validated, and quantification of a personalized risk index remains to be fully defined. Nevertheless, the ongoing progress in these areas - coupled with collaboration between centres to share structured, homogeneous data - provide plenty of reasons for optimism. In fact, we believe that the advances described in this review will place radiation oncology at the vanguard of precision medicine in breast cancer in coming years. As always, the overriding aim is to cure more patients and improve clinical outcomes.

\section{Abbreviations}

LRR: locoregional recurrence; RT-PCR: real-time PCR; RSI: radiation sensitivity index; ER: estrogen receptors; PR: progesterone receptors; HER2: human epidermal receptor 2; DCIS: ductal carcinoma in situ; ROR: risk of recurrence; FFPE: formalin-fixed, paraffin-embedded; PMRT: post-mastectomy radiotherapy; IMRT: intensity modulated radiation therapy.

\section{Acknowledgements}

This work was jointly supported by the National Natural Science Foundation of China (No.81900264), Shenzhen Science and Technology Project (JCYJ20170817172116272) and the Special Fund for Economic and Scientific Development in Longgang District, Shenzhen City, Guangdong Province (No. LGKCYLWS2019000663, No. LGKCYLWS2020043).

\section{Competing Interests}

The authors have declared that no competing interest exists.

\section{References}

1. Bray F, Ferlay J, Soerjomataram I, et al. Global cancer statistics 2018: GLOBOCAN estimates of incidence and mortality worldwide for 36 cancers in 185 countries. CA Cancer J Clin. 2018; 68: 394-424.

2. Amin MB, Greene F, Edge S, et al. The Eighth Edition AJCC Cancer Staging Manual: Continuing to build a bridge from a population-based to a more "personalized" approach to cancer staging. CA Cancer J Clin. 2017;67(2):93-99.

3. $\mathrm{Ng} \mathrm{CK}$, Schultheis AM, Bidard FC, et al. Breast cancer genomics from microarrays to massively parallel sequencing: paradigms and new insights. J Natl Cancer Inst. 2015; 107:djv015.

4. Colomer R, Aranda-López I, Albanell J, et al. Biomarkers in breast cancer: a consensus statement by the Spanish Society of Medical Oncology and the Spanish Society of Pathology. Clin Transl Oncol. 2018;20:815-826.

5. Ademuyiwa FO, Miller A, O'Connor T, et al. The effects of oncotype DX recurrence scores on chemotherapy utilization in a multi-institutional breast cancer cohort. Breast Cancer Res Treat. 2011;126:797-802.

6. Fisher B, Anderson S, Bryant J, et al. Twenty-year follow-up of a randomized trial comparing total mastectomy, lumpectomy, and lumpectomy plus irradiation for the treatment of invasive breast cancer. N Engl J Med. 2002;347:1233-1241.

7. Early breast cancer trialists' collaborative group (EBCTCG), Darby S, McGale P, et al. Effect of radiotherapy after breast-conserving surgery on 10-year recurrence and 15-year breast cancer death: meta-analysis of individual patient data for 10801 women in 17 randomised trials. Lancet. 2011;378:1707-1716.

8. Fyles AW, McCready DR, Manchul LA, et al. Tamoxifen with or without breast irradiation in women 50 years of age or older with early breast cancer. N Engl J Med. 2004;351:963-970.
9. Hughes KS, Schnaper LA, Bellon JR, et al. Lumpectomy plus tamoxifen with or without irradiation in women age 70 years or older with early breast cancer: long-term follow-up of CALGB 9343. J Clin Oncol. 2013;31:2382-2387.

10. Fowble BL, Einck JP, Kim DN, et al. Role of postmastectomy radiation after neoadjuvant chemotherapy in stage II-III breast cancer. Int J Radiat Oncol Biol Phys. 2012;83:494-503.

11. Baumann M, Krause M, Overgaard J, et al. Radiation oncology in the era of precision medicine. Nat Rev Cancer. 2016;16:234-249.

12. Jain KK. Personalized medicine. Curr Opin Mol Ther. 2002;4:548-558.

13. National Research Council (US) Committee on a framework for developing a new taxonomy of disease. Toward precision medicine: building a knowledge network for biomedical research and a new taxonomy of disease. Washington (DC), USA: National Academies Press. 2011

14. Khoury MJ, Galea S. Will precision medicine improve population health? JAMA. 2016;316:1357-1358.

15. Marks LB, Yorke ED, Jackson A, et al. Use of normal tissue complication probability models in the clinic. Int J Radiat Oncol Biol Phys. 2010;76(Suppl 3):S10-S19.

16. Byungchul Cho. Intensity-modulated radiation therapy: a review with a physics perspective. Radiat Oncol J. 2018; 36: 1-10.

17. Wang $\mathrm{H}, \mathrm{Mu} \mathrm{X}, \mathrm{He} \mathrm{H}$, et al. Cancer radiosensitizers. Trends Pharmacol Sci. 2018;39:24-48.

18. Demaria S, Coleman CN, Formenti SC. Radiotherapy: changing the game in immunotherapy. Trends Cancer. 2016;2:286-294

19. Meehan J, Gray M, Martínez-Pérez C, et al. Precision medicine and the role of biomarkers of radiotherapy response in breast cancer. Front Oncol. 2020;10:628.

20. Hall WA, Bergom C, Thompson RF, et al. Precision oncology and genomically guided radiation therapy: a report from the American Society for Radiation Oncology/American Association of Physicists in Medicine/National Cancer Institute Precision Medicine Conference. Int J Radiat Oncol Biol Phys. 2018;101:274-284

21. Braunstein LZ, Taghian AG. Molecular phenotype, multigene assays, and the locoregional management of breast cancer. Semin Radiat Oncol. 2016;26:9-16.

22. Nguyen PL, Taghian AG, Katz MS, et al. Breast cancer subtype approximated by estrogen receptor, progesterone receptor, and HER-2 is associated with local and distant recurrence after breast-conserving therapy. J Clin Oncol. 2008;26:2373-2378.

23. Caudle AS, Yu TK, Tucker SL, et al. Local-regional control according to surrogate markers of breast cancer subtypes and response to neoadjuvant chemotherapy in breast cancer patients undergoing breast conserving therapy. Breast Cancer Res. 2012; 14: R83.

24. Guarneri V, Broglio K, Kau SW, et al. Prognostic value of pathologic complete response after primary chemotherapy in relation to hormone receptor status and other factors. J Clin Oncol. 2006;24:1037-1044.

25. Esserman LJ, Berry DA, Cheang MC, et al. Chemotherapy response and recurrence-free survival in neoadjuvant breast cancer depends on biomarker profiles: results from the I-SPY 1 TRIAL (CALGB 150007/150012; ACRIN 6657). Breast Cancer Res Treat. 2012;132:1049-1062.

26. Kwa M, Makris A, Esteva FJ. Clinical utility of gene-expression signatures in early stage breast cancer. Nat Rev Clin Oncol. 2017;14:595-610.

27. Albain KS, Barlow WE, Shak S, et al. Prognostic and predictive value of the 21-gene recurrence score assay in postmenopausal women with node-positive, oestrogen-receptor-positive breast cancer on chemotherapy: a retrospective analysis of a randomised trial. Lancet Oncol. 2010;11:55-65.

28. Paik S, Shak S, Tang G, et al. A multigene assay to predict recurrence of tamoxifen-treated, node-negative breast cancer. N Engl J Med. 2004;351: 2817-2826.

29. Dowsett M, Cuzick J, Wale C, et al. Prediction of risk of distant recurrence using the 21-gene recurrence score in node-negative and node-positive postmenopausal patients with breast cancer treated with anastrozole or tamoxifen: A TransATAC study. J Clin Oncol. 2010;28:1829-1834.

30. Asad J, Jacobson AF, Estabrook A, et al. Does oncotype DX recurrence score affect the management of patients with early-stage breast cancer? Am J Surg. 2008;196:527-529.

31. Mamounas EP, Tang G, Fisher B, et al. Association between the 21-gene recurrence score assay and risk of locoregional recurrence in node-negative, estrogen receptor-positive breast cancer: Results from NSABP B-14 and NSABP B-20. J Clin Oncol. 2010;28:1677-1683

32. Fisher B, Dignam J, Wolmark N, et al. Lumpectomy and radiation therapy for the treatment of intraductal breast cancer: findings from National Surgical Adjuvant Breast and Bowel Project B-17. J Clin Oncol. 1998;16:441-452.

33. Solin LJ, Gray R, Hughes LL, et al. Surgical excision without radiation for ductal carcinoma in situ of the breast: 12-year results from the ECOG-ACRIN E5194 study. J Clin Oncol. 2015;33:3938-3944

34. Solin LJ, Gray R, Baehner FL, et al. A multigene expression assay to predict local recurrence risk for ductal carcinoma in situ of the breast. J Natl Cancer Inst. 2013:105:701-710.

35. Rakovitch E, Nofech-Mozes S, Narod SA, et al. Can we select individuals with low risk ductal carcinoma in situ (DCIS)? A population-based outcomes analysis. Breast Cancer Res Treat. 2013;138:581-590.

36. Rakovitch E, Nofech-Mozes S, Hanna W, et al. A population-based validation study of the DCIS Score predicting recurrence risk in individuals treated by breast-conserving surgery alone. Breast Cancer Res Treat. 2015;152:389-398.

37. Rakovitch E, Nofech-Mozes S, Hanna W, et al. Multigene expression assay and benefit of radiotherapy after breast conservation in ductal carcinoma in situ. J Natl Cancer Inst. 2017;109:djw256.

38. Alvarado M, Carter DL, Guenther JM, et al. The impact of genomic testing on the recommendation for radiation therapy in patients with ductal carcinoma in situ: a prospective clinical utility assessment of the 12-gene DCIS score ${ }^{\mathrm{TM}}$ result. J Surg Oncol. 2015;111:935-940.

39. Nofech-Mozes S, Hanna W, Rakovitch E. Molecular evaluation of breast ductal carcinoma in situ with Oncotype DX DCIS. Am J Pathol. 2019:189:975-980.

40. Perou CM, Sørlie T, Eisen MB, et al. Molecular portraits of human breast tumours. Nature. 2000;406:747-752 
41. Sorlie T, Tibshirani R, Parker J, et al. Repeated observation of breast tumor subtypes in independent gene expression data sets. Proc Natl Acad Sci USA. 2003;100:8418-8423

42. Parker JS, Mullins M, Cheang MC, et al. Supervised risk predictor of breast cancer based on intrinsic subtypes. J Clin Oncol. 2009;27:1160-1167.

43. Bastien RR, Rodríguez-Lescure Á, Ebbert MT, et al. PAM50 breast cancer subtyping by RT-qPCR and concordance with standard clinical molecular markers. BMC Med Genomics. 2012;5:44.

44. Dowsett M, Sestak I, Lopez-Knowles E, et al. Comparison of PAM50 risk of recurrence score with oncotype DX and IHC4 for predicting risk of distant recurrence after endocrine therapy. J Clin Oncol. 2013:31:2783-2790.

45. Gnant M, Filipits M, Greil R, et al. Predicting distant recurrence in receptorpositive breast cancer patients with limited clinicopathological risk: using the PAM50 risk of recurrence score in 1478 postmenopausal patients of the ABCSG-8 trial treated with adjuvant endocrine therapy alone. Ann Oncol. 2014;25:339-345.

46. Fitzal F, Filipits M, Fesl C, et al. Predicting local recurrence using PAM50 in postmenopausal endocrine responsive breast cancer patients. J Clin Oncol. 2014 32(Suppl 15):1008

47. Overgaard $\mathrm{M}$, Jensen $\mathrm{MB}$, Overgaard J, et al. Postoperative radiotherapy in high-risk postmenopausal breast-cancer patients given adjuvant tamoxifen: Danish breast cancer cooperative group DBCG 82c randomised trial. Lancet. 1999:353:1641-1648.

48. Tramm T, Mohammed H, Myhre S, et al. Development and validation of a gene profile predicting benefit of postmastectomy radiotherapy in patients with high-risk breast cancer: a study of gene expression in the DBCG82bc cohort. Clin Cancer Res. 2014;20:5272-5280.

49. Eschrich $\mathrm{S}$, Zhang $\mathrm{H}$, Zhao $\mathrm{H}$, et al. Systems biology modeling of the radiation sensitivity network: a biomarker discovery platform. Int J Radiat Oncol Biol Phys. 2009;75:497-505.

50. Eschrich SA, Fulp WJ, Pawitan Y, et al. Validation of a radiosensitivity molecular signature in breast cancer. Clin Cancer Res. 2012;18:5134-5143.

51. Sjöström M, Staaf J, Edén P, et al. Identification and validation of single-sample breast cancer radiosensitivity gene expression predictors. Breast Cancer Res. 2018;20:64.

52. Torres-Roca JF, Fulp WJ, Caudell JJ, et al. Integration of a radiosensitivity molecular signature into the assessment of local recurrence risk in breast cancer. Int J Radiat Oncol Biol Phys. 2015;93:631-638.

53. McMahon SJ. The linear quadratic model: usage, interpretation and challenges. Phys Med Biol. 2018;64:01TR01.

54. Scott JG, Berglund A, Schell MJ, et al. A genome-based model for adjusting radiotherapy dose (GARD): a retrospective, cohort-based study. Lancet Oncol. 2017:18:202-211.

55. Poortmans P, Kaidar-Person O, Span P. Radiation oncology enters the era of individualised medicine. Lancet Oncol. 2017;18:159-160

56. Ahmed KA, Liveringhouse CL, Mills MN, et al. Utilizing the genomically adjusted radiation dose (GARD) to personalize adjuvant radiotherapy in triple negative breast cancer management. EBioMedicine. 2019;47:163-169.

57. Kraus-Tiefenbacher U, Sfintizky A, Welzel G, et al. Factors of influence on acute skin toxicity of breast cancer patients treated with standard threedimensional conformal radiotherapy (3D-CRT) after breast conserving surgery (BCS). Radiat Oncol. 2012;7:217.

58. Chan TY, Tang JI, Tan PW, et al. Dosimetric evaluation and systematic review of radiation therapy techniques for early stage node-negative breast cancer treatment. Cancer Manag Res. 2018:10:4853-4870.

59. Mukesh MB, Barnett GC, Wilkinson JS, et al. Randomized controlled trial of intensity-modulated radiotherapy for early breast cancer: 5-year results confirm superior overall cosmesis. J Clin Oncol. 2013;31:4488-4495.

60. Korzets Y, Fyles A, Shepshelovich D, et al. Toxicity and clinical outcomes of partial breast irradiation (PBI) compared to whole breast irradiation (WBI) for early stage breast cancer: a systematic review and meta-analysis. Breast Cancer Res Treat. 2019;175:531-545.

61. Safwat A, Bentzen SM, Turesson I, et al. Deterministic rather than stochastic factors explain most of the variation in the expression of skin telangiectasia after radiotherapy. Int J Radiat Oncol Biol Phys. 2002;52:198-204.

62. Barnett GC, Coles CE, Elliott RM, et al. Independent validation of genes and polymorphisms reported to be associated with radiation toxicity: a prospective analysis study. Lancet Oncol. 2012;13:65-77.

63. Turnbull C, Ahmed S, Morrison J, et al. Genome-wide association study identifies five new breast cancer susceptibility loci. Nat Genet. 2010:42:504-507.

64. Fachal L, Dunning AM. From candidate gene studies to GWAS and post-GWAS analyses in breast cancer. Curr Opin Genet Dev. 2015;30:32-41

65. Matuszak MM, Fuller CD, Yock TI, et al. Performance/outcomes data and physician process challenges for practical big data efforts in radiation oncology. Med Phys. 2018:45:e811-e819.

66. Andreassen $\mathrm{CN}$. Searching for genetic determinants of normal tissue radiosensitivity --Are we on the right track? Radiother Oncol. 2010;97:1-8.

67. Talbot CJ, Tanteles GA, Barnett GC, et al. A replicated association between polymorphisms near TNFa and risk for adverse reactions to radiotherapy. $\mathrm{Br} \mathrm{J}$ Cancer. 2012;107:748-753

68. Kerns SL, Fachal L, Dorling L, et al. Radiogenomics consortium genome-wide association study meta-analysis of late toxicity after prostate cancer radiotherapy. J Natl Cancer Inst. 2020;112:179-190.

69. Burnet NG, Barnett GC, Summersgill HR, et al. RAPPER-a success story for collaborative translational radiotherapy research. Clin Oncol. 2019;31:416-419.

70. Kerns SL, de Ruysscher D, Andreassen CN, et al. STROGAR-strengthening the reporting of genetic association studies in radiogenomics. Radiother Oncol. 2014;110:182-188.

71. van Gent DC, Hoeijmakers JH, Kanaar R. Chromosomal stability and the DNA double-stranded break connection. Nat Rev Genet. 2001;2:196-206.

72. Pollard JM, Gatti RA. Clinical radiation sensitivity with DNA repair disorders: an overview. Int J Radiat Oncol Biol Phys. 2009;74:1323-1331.
73. Zhang Y, Liu Z, Wang M, et al. Single nucleotide polymorphism rs1801516 in ataxia telangiectasia-mutated gene predicts late fibrosis in cancer patients after radiotherapy. Medicine. 2016;95:e3267.

74. Dong L, Cui J, Tang F, et al. Ataxia telangiectasia-mutated gene polymorphisms and acute normal tissue injuries in cancer patients after radiation therapy: a systematic review and meta-analysis. Int J Radiat Oncol Biol Phys. 2015;91:1090-1098.

75. Su M, Yin ZH, Wu W, et al. Meta-analysis of associations between ATM Asp1853Asn and TP53 Arg72Pro polymorphisms and adverse effects of cancer radiotherapy. Asian Pac J Cancer Prev. 2014;15:10675-10681.

76. Andreassen $\mathrm{CN}$, Rosenstein BS, Kerns SL, et al. Individual patient data metaanalysis shows a significant association between the ATM rs1801516 SNP and toxicity after radiotherapy in 5456 breast and prostate cancer patients. Radiother Oncol. 2016;121:431-439.

77. Lee E, Takita C, Wright JL, et al. Genome-wide enriched pathway analysis of acute post-radiotherapy pain in breast cancer patients: a prospective cohort study. Hum Genomics. 2019:13:28.

78. Córdoba EE, Abba MC, Lacunza E, et al. Polymorphic variants in oxidative stress genes and acute toxicity in breast cancer patients receiving radiotherapy. Cancer Res Treat. 2016;48:948-954.

79. Grossberg $\mathrm{AJ}$, Lei $\mathrm{X}, \mathrm{Xu} \mathrm{T}$, et al. Association of transforming growth factor $\beta$ polymorphism C-509T with radiation-induced fibrosis among patients with early-stage breast cancer: a secondary analysis of a randomized clinical trial. JAMA Oncol. 2018;4:1751-1757.

80. Haviland JS, Owen JR, Dewar JA, et al. The UK standardisation of breast radiotherapy (START) trials of radiotherapy hypofractionation for treatment of early breast cancer: 10-year follow-up results of two randomised controlled trials. Lancet Oncol. 2013;14:1086-1094.

81. Smith BD, Arthur DW, Buchholz TA, et al. Accelerated partial breast irradiation consensus statement from the American society for radiation oncology (ASTRO). Int J Radiat Oncol Biol Phys. 2009;74:987-1001.

82. Herskovic $\mathrm{AC}, \mathrm{Wu} \mathrm{X}, \mathrm{Christos} \mathrm{PJ}$, et al. Omission of adjuvant radiotherapy in the elderly breast cancer patient: missed opportunity? Clin Breast Cancer. 2018;18:418-431.

83. Sakorafas GH, Safioleas M. Breast cancer surgery: an historical narrative. Part III. from the sunset of the 19th to the dawn of the 21st century. Eur J Cancer Care. 2010;19:145-166.

84. [Internet] NSABP Foundation. A randomized phase III clinical trial evaluating post-mastectomy chestwall and regional nodal XRT and post-lumpectomy regional nodal XRT in patients with positive axillary nodes before neoadjuvant chemotherapy who convert to pathologically negative axillary nodes after neoadjuvant chemotherapy. https://clinicaltrials.gov/ct2/show/NCT01872975.

85. Ow SG, Lee SC. Precision medicine in early breast cancer-can this apply to radiotherapy? J Thorac Dis. 2016;8:2982-2985.

\section{Author Biography}

Dr. Jianshe Yang is a professor of radiotherapy and cancer biology at $3^{\text {rd }}$ Affiliated Hospital, the Chinese University of Hongkong (Shenzhen). He obtained Ph.D. of radiobiology at Institute of Modern Physics, Chinese Academy of Sciences in 2005, and worked as postdoctoral fellow at Shanghai Institute of Applied Physics, Chinese Academy of Sciences from March 2006 to March 2008. He has authored and coauthored over 100 publications including New England Journal of Medicine, Carbon, Science in China, Radiation and Environmental Biophysics, and Advances in Space Research. He holds 5 China patents. The current research interests in Professor Yang's group include: (1) Radiotherapy to solid tumors by accelerated heavy charged particles; (2) Radiobioeffects and radiosensitizer of nanomaterials; and (3) Development of nanomaterials-based anti-coronavirus drug design. 